\title{
Matched Drawings of Planar Graphs ${ }^{\star}$
}

\author{
Emilio Di Giacomo ${ }^{1}$, Walter Didimo ${ }^{1}$, Marc van Kreveld ${ }^{2}$, Giuseppe Liotta ${ }^{1}$, \\ and Bettina Speckmann ${ }^{3}$
}

${ }^{1}$ Dip. di Ingegneria Elettronica e dell'Informazione, Università degli Studi di Perugia \{digiacomo, didimo, liotta\}@diei.unipg.it

2 Department of Computer Science, Utrecht University

marc@cs.uu.nl

${ }^{3}$ Department of Mathematics and Computer Science, TU Eindhoven

speckman@win.tue.nl

\begin{abstract}
A natural way to draw two planar graphs whose vertex sets are matched is to assign each matched pair a unique $y$-coordinate. In this paper we introduce the concept of such matched drawings, which are a relaxation of simultaneous geometric embeddings with mapping. We study which classes of graphs allow matched drawings and show that $(i)$ two 3-connected planar graphs or a 3-connected planar graph and a tree may not be matched drawable, while $(i i)$ two trees or a planar graph and a planar graph of some special families - such as unlabeled level planar (ULP) graphs or the family of "carousel graphs" - are always matched drawable.
\end{abstract}

\section{Introduction}

The visual comparison of two graphs whose vertex sets are associated in some way requires drawings of these graphs that highlight their association in a clear manner. Drawings of this type are of use for various areas of computer science, including bio-informatics, web data mining, network analysis, and software engineering. Of course each drawing individually should be as clear as possible, using, for example, few bends and crossings. But, most importantly, the positions of associated vertices in the two drawings should be "close". This makes it possible for the user to easily identify structurally identical and structurally different portions of the two graphs, or to maintain her "mental map" [17. Structural changes between two graphs and their visualizations arise, for example, when collapsing or expanding clusters in clustered drawings, during the navigation of very large graphs with a topological window, in the analysis of the evolving relationships among the actors of a social network, and in the comparison of multiple gene trees (see, for example, [16/7/1|14|16 18]).

Two positions are definitely "close" if they are identical. Hence a substantial research effort has recently been devoted to the problem of computing straightline drawings of two graphs on the same set of points. More specifically, assume

^ Research partially supported by the MIUR Project "MAINSTREAM: Algorithms for massive information structures and data streams". 
we are given two planar graphs $G_{1}=\left(V_{1}, E_{1}\right)$ and $G_{2}=\left(V_{2}, E_{2}\right)$ with $\left|V_{1}\right|=\left|V_{2}\right|$, together with a one-to-one mapping between their vertices. A simultaneous geometric embedding with mapping (introduced by Brass et al. in [3]) of $G_{1}$ and $G_{2}$ is a pair of straight-line planar drawings $\Gamma_{1}$ and $\Gamma_{2}$ of $G_{1}$ and $G_{2}$, respectively, such that for any pair of matched vertices $u \in V_{1}$ and $v \in V_{2}$ the position of $u$ in $\Gamma_{1}$ is the same as the position of $v$ in $\Gamma_{2}$. Unfortunately, only pairs of graphs belonging to restricted subclasses of planar graphs admit a simultaneous geometric embedding with mapping. Brass et al. 3] showed how to simultaneously embed pairs of paths, pairs of cycles, and pairs of caterpillars, but they also proved that a path and a graph or two outerplanar graphs may not admit this type of drawing. Geyer, Kaufmann, and Vrt'o 15. recently proved that even a pair of trees may not have a simultaneous geometric embedding with mapping. These negative results motivated the study of relaxations of simultaneous geometric embeddings. One possibility is to introduce bends along the edges [4813, another, to allow that the same vertex occupies different locations in the two drawings [23], introducing ambiguity in the mapping.

In this paper we consider a different interpretation of two positions being "close". Instead of requiring that matched vertices occupy the same location, we assign each matched pair a unique $y$-coordinate. This enables the user to unambiguously identify pairs of matched vertices but, at the same time, leaves us more freedom to draw both graphs clearly. Specifically, let again $G_{1}=\left(V_{1}, E_{1}\right)$ and $G_{2}=\left(V_{2}, E_{2}\right)$ be two planar graphs with $\left|V_{1}\right|=\left|V_{2}\right| . G_{1}$ and $G_{2}$ are matched if there is a one-to-one mapping between $V_{1}$ and $V_{2}$. If a vertex $u \in V_{1}$ is matched with a vertex $v \in V_{2}$ then we say that $u$ is the partner of $v$ and that $v$ is the partner of $u$. A matched drawing of $G_{1}$ and $G_{2}$ is a pair of straight-line planar drawings $\Gamma_{1}$ and $\Gamma_{2}$ of $G_{1}$ and $G_{2}$, respectively, such that for any pair of matched vertices $u \in V_{1}$ and $v \in V_{2}$ the $y$-coordinate of $u$ in $\Gamma_{1}$ is the same as the $y$ coordinate of $v$ in $\Gamma_{2}$, and this $y$-coordinate is unique. If two matched graphs have a matched drawing, then we say that they are matched drawable. Matched drawings can be viewed as a relaxation of simultaneous geometric embedding with mapping. An example of a matched drawing of two trees is shown in Fig. 1.

Results and Organization. We start by presenting pairs of graphs that are not matched drawable. In particular, in Section 2.1 we describe two isomorphic 3 -connected planar graphs that both have 12 vertices and that are not matched

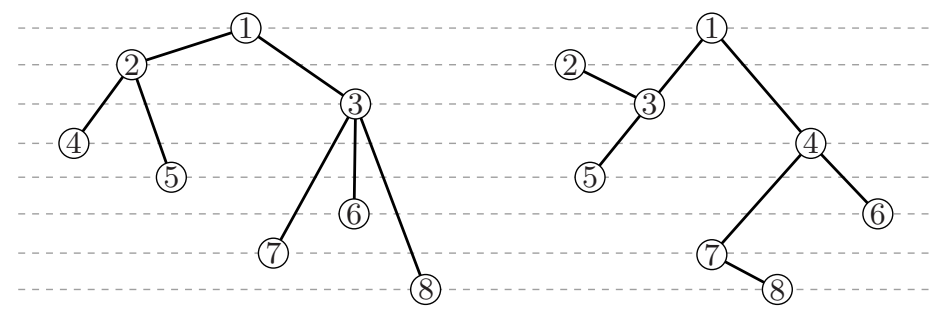

Fig. 1. A matched drawing of two trees 
drawable. We also present a 3-connected planar graph and a tree that both have 620 vertices and that are not matched drawable. This construction can be found in Section 2.2

We continue by describing drawing algorithms for classes of graphs that are always matched drawable. In particular, in Section 3.1 we show that a planar graph and an unlabeled level planar (ULP) graph that are matched are always matched drawable. In Section 3.2 we extend these results to a planar graph and a graph of the family of "carousel graphs". Finally, in Section 3.3 we prove that two matched trees are always matched drawable.

\section{Graphs That Are Not Matched Drawable}

\subsection{Two 3-Connected Graphs}

We start by stating a simple property of planar straight-line drawings.

Property 1. Let $G$ be an embedded planar graph and let $\Gamma$ be a straight-line planar drawing of $G$. Let $u$ be the vertex of $G$ with the highest $y$-coordinate in $\Gamma$ and let $v$ be the vertex of $G$ with the lowest $y$-coordinate in $\Gamma$. Vertices $u$ and $v$ belong to the external face of $G$.

Now assume that $G_{1}$ and $G_{2}$ are two matched graphs with the following properties: $(i) G_{1}$ contains two vertex-disjoint simple cycles $C_{1}=\left\{u_{1}, \ldots, u_{n}\right\}$ and $C_{1}^{\prime}=\left\{u_{1}^{\prime}, \ldots, u_{m}^{\prime}\right\},(i i) G_{2}$ contains two vertex-disjoint simple cycles $C_{2}=$ $\left\{v_{1}, \ldots, v_{n}\right\}$ and $C_{2}^{\prime}=\left\{v_{1}^{\prime}, \ldots, v_{m}^{\prime}\right\}$, and $(i i i) u_{i}$ is the partner of $v_{i}(1 \leq i \leq n)$ and $u_{j}^{\prime}$ is the partner of $v_{j}^{\prime}(1 \leq j \leq m)$. If $\Psi_{1}$ is a planar embedding of $G_{1}$ such that $C_{1}^{\prime}$ is inside $C_{1}$ and if $\Psi_{2}$ is a planar embedding of $G_{2}$ such that $C_{2}$ is inside $C_{2}^{\prime}$, then we call $\Psi_{1}$ and $\Psi_{2}$ interlaced embeddings and $C_{1}, C_{1}^{\prime}, C_{2}$, and $C_{2}^{\prime}$ interlaced cycles.

Lemma 1. Let $G_{1}$ and $G_{2}$ be two matched graphs with interlaced embeddings $\Psi_{1}$ and $\Psi_{2}$. There is no matched drawing $\Gamma_{1}$ and $\Gamma_{2}$ of $G_{1}$ and $G_{2}$ such that $\Gamma_{1}$ preserves $\Psi_{1}$ and $\Gamma_{2}$ preserves $\Psi_{2}$.

Proof. Denote by $C_{1}, C_{1}^{\prime}, C_{2}$, and $C_{2}^{\prime}$ the interlaced cycles of $\Psi_{1}$ and $\Psi_{2}$. Suppose by contradiction that $\Gamma_{1}$ and $\Gamma_{2}$ exist. Denote by $\overline{\Gamma_{1}}$ the subdrawing of $\Gamma_{1}$ restricted to the subgraph $C_{1} \cup C_{1}^{\prime}$ and by $\overline{\Gamma_{2}}$ the subdrawing of $\Gamma_{2}$ restricted to the subgraph $C_{2} \cup C_{2}^{\prime}$.

Since in $\Psi_{1}$ cycle $C_{1}^{\prime}$ is inside cycle $C_{1}$, by Property 1 the top-most and the bottom-most vertices of $\overline{\Gamma_{1}}$ belong to $C_{1}$; denote these two vertices by $u_{t}$ and $u_{b}$. Since $\overline{\Gamma_{1}}$ is planar and since the drawing of $C_{1}^{\prime}$ is completely inside the drawing of $C_{1}$, every vertex $u_{j}^{\prime}$ of $C_{1}^{\prime}$ has a $y$-coordinate that is greater than the $y$-coordinate of $u_{b}$ and smaller than the $y$-coordinate of $u_{t}$. Since $\Gamma_{1}$ and $\Gamma_{2}$ are matched drawings, every vertex $v_{j}^{\prime}$ of $C_{2}^{\prime}$ in $\overline{\Gamma_{2}}$ has a $y$-coordinate that is greater than the $y$-coordinate of $v_{b}$ (i.e., the partner of $u_{b}$ ) and smaller than the $y$-coordinate of $v_{t}$ (i.e., the partner of $u_{t}$ ). However, since in $\Psi_{2}$ cycle $C_{2}$ is inside cycle $C_{2}^{\prime}$, by Property 1 the top-most and the bottom-most vertices of $\overline{\Gamma_{2}}$ belong to $C_{2}^{\prime}$, a contradiction. 

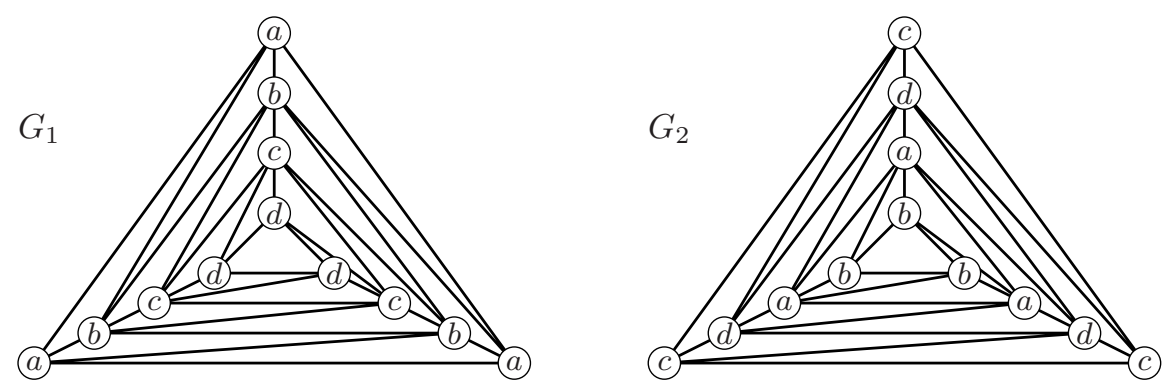

Fig. 2. Two 3-connected planar graphs that are not matched drawable. The partner of a vertex of $G_{1}$ is any vertex in $G_{2}$ that has the same label.

Theorem 1. There exist two 3-connected planar graphs that are not matched drawable.

Proof (sketch). Consider the two 3-connected planar graphs $G_{1}$ and $G_{2}$ in Fig. 2 The partner of a vertex of $G_{1}$ is any vertex in $G_{2}$ that has the same label. To prove that $G_{1}$ and $G_{2}$ are not matched drawable, we show that all planar embeddings of $G_{1}$ and $G_{2}$ are interlaced embeddings. The proof uses a case analysis on the choice of the external faces and is omitted for reasons of space.

\subsection{A 3-Connected Graph and a Tree}

The two graphs described in Theorem 1 are both 3 -connected. Hence the question arises if two planar graphs, at least one of which is not 3-connected, are always matched drawable. This is unfortunately not the case: in the following we present a planar graph and a tree that are not matched drawable.

Given a vertex $v$ of a graph $G$ and a drawing $\Gamma$ of $G$, we denote by $x(v)$ and $y(v)$ the $x$ - and $y$-coordinate of $v$ in $\Gamma$. Let $T^{*}=\left(V^{*}, E^{*}\right)$ be the tree depicted in Fig. 3. Estrella-Balderrama et al. 10] proved the following lemma:

Lemma 2 (Estrella-Balderrama et al. [10]). Let $T^{*}$ be the tree depicted in Fig. 3. A straight-line planar drawing $\Gamma$ of $T^{*}$ such that $y\left(v_{0}\right)<y\left(v_{7}\right)<y\left(v_{3}\right)<$ $y\left(v_{2}\right)<y\left(v_{4}\right)<y\left(v_{1}\right)<y\left(v_{5}\right)<y\left(v_{6}\right)$ in $\Gamma$ does not exist.

Let $T^{*}$ be rooted at vertex $v_{0}$, and for each vertex $v_{i}$, denote by $d\left(v_{i}\right)$ the graphtheoretic distance of $v_{i}$ from the root $(i=0,1, \ldots, 7)$. We construct a tree $T$ by using $T^{*}$ as a model. $T$ has $3^{d\left(v_{i}\right)}$ copies of each vertex $v_{i}(i=0,1, \ldots, 7)$. The $3^{d\left(v_{i}\right)}$ copies of $v_{i}$ are denoted as $v_{i, 0}, v_{i, 1}, \ldots, v_{i, 3^{d\left(v_{i}\right)}-1}$. Vertex $v_{h, k}$ is a child of vertex $v_{i, j}$ in $T$ if and only if $v_{h}$ is a child of $v_{i}$ in $T^{*}$ and $j=\lfloor k / 3\rfloor$ $(0 \leq i, h \leq 7),\left(0 \leq j \leq 3^{d\left(v_{i}\right)}-1\right),\left(0 \leq k \leq 3^{d\left(v_{h}\right)}-1\right)$. So $T$ has one copy of $v_{0}$ whose children are the three copies $v_{1,0}, v_{1,1}$, and $v_{1,2}$ of $v_{1}$. The children of each copy of $v_{1}$ are three of the nine copies of $v_{2}$, and so on. Three vertices of $T$ with the same parent are called a triplet of $T$. The total number of vertices of $T$ is 310 . 


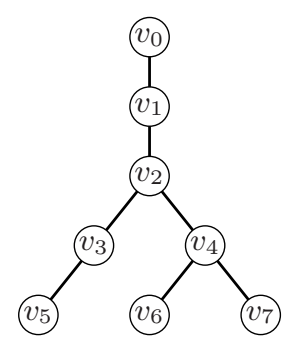

Fig. 3. A tree that does not have a straight-line planar drawing with $y\left(v_{0}\right)<y\left(v_{7}\right)<y\left(v_{3}\right)<$ $y\left(v_{2}\right)<y\left(v_{4}\right)<y\left(v_{1}\right)<y\left(v_{5}\right)<y\left(v_{6}\right)$ 10
Table 1. Matching between the vertices of $T$ and the vertices of $G_{103}$

\begin{tabular}{|c|c|c|c|}
\hline vertex & copies & triplets & levels \\
\hline \hline$v_{7}$ & 81 & 27 & $1 \ldots 27$ \\
\hline$v_{3}$ & 27 & 9 & $28 \ldots 36$ \\
\hline$v_{2}$ & 9 & 3 & $37 \ldots 39$ \\
\hline$v_{4}$ & 27 & 9 & $40 \ldots 48$ \\
\hline$v_{1}$ & 3 & 1 & 49 \\
\hline$v_{5}$ & 81 & 27 & $50 \ldots 76$ \\
\hline$v_{6}$ & 81 & 27 & $77 \ldots 103$ \\
\hline
\end{tabular}

The tree $T$ is matched with a nested-triangles graph, which is defined as follows. A single vertex $v$ is a nested-triangles graph denoted by $G_{0}$. A triangulated planar embedded graph $G_{k}(k>0)$ is a nested-triangles graph if the external face of $G_{k}$ has exactly three vertices and the graph $G_{k-1}$, obtained by removing the vertices on the external face, is still a nested-triangles graph. A levelling of the vertices is naturally defined for the vertices of $G_{k}$ : level $i$ of $G_{k}$ contains the vertices that are on the external face of $G_{i}(i=0,1, \ldots, k)$. Note that $G_{k}$ has $3 k+1$ vertices and $k+1$ levels. Thus, $G_{103}$ has 310 vertices and 104 levels.

$T$ and $G_{103}$ are matched in the following way. Vertex $v_{0}$ is mapped to the (only) vertex of level 0 . Each triplet of $T$ is mapped to three vertices of $G_{103}$ such that the level of these three vertices is the same in $G_{103}$. Also, all triplets formed by vertices that are copies of the same vertex of $T^{*}$ are mapped to consecutive levels of $G_{103}$. The exact mapping is described in Table 1 Each row of the table refers to a different vertex of $T^{*}$ and shows the number of copies of that vertex in $T$, the number of triplets in $T$, and the levels of $G_{103}$ to which these triplets are mapped (a triplet for each level).

We now prove that, with the mapping described by Table [1] $T$ and $G_{103}$ are not matched drawable if we insist that the drawing of $G_{103}$ preserves the embedding of $G_{103}$. We start with a useful property.

Property 2. Let $\Gamma_{G_{103}}$ be any planar straight-line drawing of $G_{103}$ that preserves the embedding of $G_{103}$. For each level $i(0 \leq i \leq 103)$ there exists a vertex of level $i$ that has $y$-coordinate greater than the $y$-coordinates of all the vertices having level less than $i$.

Lemma 3. A matched drawing $\Gamma_{T}$ and $\Gamma_{G_{103}}$ of the tree $T$ and the graph $G_{103}$ such that $\Gamma_{G_{103}}$ preserves the embedding of $G_{103}$ does not exist.

Proof (sketch). Let $\Gamma_{G_{103}}$ be any planar straight-line drawing of $G_{103}$ that preserves the embedding of $G_{103}$. By exploiting Property 2 , we can show that $\Gamma_{G_{103}}$ induces an ordering $\lambda$ of the vertices of $T$ along the $y$-direction such that there exists a subtree $T^{\prime}$ of $T$ isomorphic to $T^{*}$ for which the ordering $\lambda$ restricted to the vertices of $T^{\prime}$ is the ordering given in Lemma 2 (the proof about how $T^{\prime}$ is 
defined is omitted). This implies that $T^{\prime}$ (and hence $T$ ) does not have a planar straight-line drawing that respects the ordering induced by $\Gamma_{G_{103}}$.

According to Lemma 3. $T$ and $G_{103}$ are not matched drawable in the case that one wants a drawing of $G_{103}$ that preserves the embedding of $G_{103}$. In the following theorem we show that $T$ and $G_{103}$ can be used to construct a new tree and a new 3-connected planar graph that are not matched drawable even if we allow the embedding to be changed.

Theorem 2. There exist a tree and a 3-connected planar graph that are not matched drawable.

Proof (sketch). Let $\bar{T}$ be a tree that consists of two copies of $T$ whose roots are adjacent. Let $G$ be a graph obtained by taking two distinct copies of $G_{103}$ and connecting the vertices of their external faces in such a way that the obtained graph is a triangulated planar graph. The matching of the vertices is such that a copy of $T$ matches a copy of $G_{103}$ as before. We observe that any embedding of $G$ leaves one of the copies of $G_{103}$ as in Lemma 3 .

\section{Matched Drawable Graphs}

In this section we describe drawing algorithms for classes of graphs that are always matched drawable. In particular, in Section 3.1 we show that a planar graph and an unlabeled level planar (ULP) graph that are matched are always matched drawable. In Section 3.2 we extend these results to a planar graph and a graph of the family of "carousel graphs". Finally, in Section 3.3 we prove that two matched trees are always matched drawable.

These results show that matched drawings do indeed allow larger classes of graphs to be drawn than simultaneous geometric embeddings with mapping (a path and a planar graph may not admit a simultaneous geometric embedding with mapping [3] and the same negative result also holds for pairs of trees [15]).

\subsection{Planar Graphs and ULP Graphs}

ULP graphs were defined by Estrella-Balderrama, Fowler, and Kobourov [10]. Let $G$ be a planar graph with $n$ vertices. A $y$-assignment of the vertices of $G$ is a one-to-one mapping $\lambda: V \rightarrow \mathbb{N}$. A drawing of $G$ compatible with $\lambda$ is a planar straight-line drawing of $G$ such that $y(v)=\lambda(v)$ for each vertex $v \in V$. A planar graph $G$ is unlabeled level planar (ULP) if for any given $y$-assignment $\lambda$ of its vertices, $G$ admits a drawing compatible with $\lambda$.

Theorem 3. A planar graph and an ULP graph are always matched drawable.

Proof (sketch). Let $G_{1}$ be a planar graph and let $G_{2}$ be an ULP graph. Compute a planar straight-line drawing of $G_{1}$ such that each vertex has a different $y$ coordinate, for example with a slight variant of the algorithm of de Fraysseix, Pach, and Pollack [5]. The drawing of $G_{1}$ together with the mapping between 
$G_{1}$ and $G_{2}$ defines a $y$-assignment $\lambda$ for $G_{2}$. Since $G_{2}$ is ULP it admits a drawing compatible with $\lambda$. It follows that $G_{1}$ and $G_{2}$ are matched drawable.

ULP trees are characterized in [10]. A complete characterization of ULP graphs has very recently been given in [12. A planar graph is ULP if and only if it is either a generalized caterpillar, or a radius-2 star, or a generalized degree-3 spider. These graphs are defined as follows (see also [12). A graph is a caterpillar if deleting all vertices of degree one produces a path, which is called the spine of the caterpillar. A generalized caterpillar is a graph that contains cycles of length at most 4 in which every spanning tree is a caterpillar such that no three cut vertices are pairwise adjacent and no pair of adjacent cut vertices belong to the same 4-cycle. A radius-2 star is a $K_{1, k}, k>2$, in which every edge is subdivided at most once. The only vertex of degree $k$ is called the center of the star. A degree-3 spider is an arbitrary subdivision of $K_{1,3}$. A generalized degree-3 spider is a graph with maximum degree 3 in which every spanning tree is either a path or a degree-3 spider.

Corollary 1. Let $G_{1}$ and $G_{2}$ be two matched graphs such that $G_{1}$ is a planar graph and $G_{2}$ is either a generalized caterpillar, or a radius-2 star, or a generalized degree-3 spider. Then $G_{1}$ and $G_{2}$ are matched drawable.

\subsection{Planar Graphs and Carousel Graphs}

In this section we extend the result of Theorem 3 by describing a family of graphs that also includes non-ULP graphs and whose members have a matched drawing with any planar graph. Let $G$ be a planar graph, let $v$ be a vertex of $G$, and let $\Gamma$ be a planar straight-line drawing of $G$. $\Gamma$ is $v$-stretchable if: $(i)$ there is a vertical ray from $v$ going to $+\infty$ that does not intersect any edge of $\Gamma$, and $(i i)$ for any given $\Delta>0$, there exists a value $\Delta^{\prime} \geq \Delta$ such that the drawing obtained by translating each vertex $u$ with $x(u) \geq x(v)$ to point $\left(x(u)+\Delta^{\prime}, y(u)\right)$ is still planar. Graph $G$ is ULP $v$-stretchable if for every given $y$-assignment $\lambda$ of its vertices, $G$ admits a $v$-stretchable drawing compatible with $\lambda$.

A carousel graph is a connected planar graph $G$ consisting of a vertex $v_{0}$, called the pivot of $G$, and of a set of disjoint subgraphs $S_{1}, \ldots, S_{k}(k>1)$ such that each $S_{i}$ has a single vertex $v_{i}$ adjacent to $v_{0}(i=1, \ldots, k)$ and $S_{i}$ is ULP $v_{i}$-stretchable. Each subgraph $S_{i}$ is called a seat of $G$. Vertex $v_{i}$ is called the hook of $S_{i}$.

Theorem 4. Any planar graph and any carousel graph that are matched are always matched drawable.

Proof. Let $G_{1}$ be a planar graph and let $G_{2}$ by a carousel graph. Let $v_{0}$ be the pivot of $G_{2}$ and let $u$ be the partner of $v_{0}$ in $G_{1}$. Compute a planar straight-line drawing of $G_{1}$ such that all vertices have different $y$-coordinates and $u$ has the largest $y$-coordinate. The drawing of $G_{1}$ together with the mapping between $G_{1}$ and $G_{2}$ defines a $y$-assignment $\lambda$ for $G_{2}$. Clearly $\lambda(w)<\lambda\left(v_{0}\right)=y_{M}$ for all vertices $w \neq v_{0}$ of $G_{2}$. 
In the following we describe an incremental method to compute a drawing of $G_{2}$ compatible with $\lambda$. Let $S_{1}, \ldots, S_{k}(k>1)$ be the seats of $G_{2}$ and let $v_{i}$ be the hook of $S_{i}(1 \leq i \leq k)$. Let $\lambda_{i}$ be the $y$-assignment of the vertices of $S_{i}$ induced by $\lambda$. As a preliminary step we compute a drawing $\Gamma_{i}$ for each $S_{i}$ that is compatible with $\lambda_{i}$ and that is $v_{i}$-stretchable. Such a drawing exists because $S_{i}$ is ULP $v_{i}$-stretchable. We further assume that the distance between any two different $x$-coordinates is at least 1 unit.

We initialize the drawing by placing $v_{0}$ at position $\left(0, y_{M}\right)$, which results in drawing $\Gamma_{2}^{0}$. Drawing $\Gamma_{2}^{i}$ is constructed from drawing $\Gamma_{2}^{i-1}$ by adding drawing $\Gamma_{i}$ at a suitable $x$-location and possibly translating some of its vertices further in $x$-direction (see Fig. 4). Hence the final drawing respects $\lambda$.

Let $\mathcal{R}_{i-1}$ be the bounding box of $\Gamma_{2}^{i-1}$ and let $\left(x_{M}, y_{M}\right)$ be the coordinates of its top-right corner. Further let $R_{i}$ be the bounding box of $\Gamma_{i}$. Place the drawing $\Gamma_{i}$ such that the left side of $R_{i}$ is contained in the vertical line $x=x_{M}+1$. Let $R_{i}^{\prime}$ be the (possibly empty) sub-rectangle of $R_{i}$ delimited by the $x$-coordinates $x_{M}+1$ and $x_{M}^{\prime}=x\left(v_{i}\right)-1$. Further let $y_{M}^{\prime}$ denote the maximum $y$-coordinate of any vertex of $\Gamma_{2}^{i-1}$ or $\Gamma_{i}$ different from $v_{0}$ and let $p=\left(x_{M}^{\prime}+1, y_{M}^{\prime}\right)$. The line $\ell$ through $v_{0}$ and $p$ crosses neither $\Gamma_{2}^{i-1}$ nor the portion of $\Gamma_{i}$ contained in $R_{i}^{\prime}$ (see Fig. 4(a)). Let $q$ denote the intersection of $\ell$ with the horizontal line at $y\left(v_{i}\right)$ and let $\Delta=x(q)-x\left(v_{i}\right)$. Since $\Gamma_{i}$ is $v_{i}$-stretchable, there exists a value $\Delta^{\prime} \geq \Delta$ such that we can translate the portion of $\Gamma_{i}$ contained in $R_{i} \backslash R_{i}^{\prime}$ to the right by $\Delta^{\prime}$ without creating any crossing (see Fig. 4(b)). It can easily be verified that we can now connect $v_{i}$ to $v_{0}$ without creating any crossings.

Lemma 4. Let $G$ be a simple cycle and let $v$ be any vertex of $G$. $G$ is ULP $v$-stretchable.

Proof. Let $\lambda$ be any $y$-assignment of the vertices of $G$ and let $u$ be the vertex of $G$ that has the smallest $y$-coordinate. Let $u=v_{0}, v_{1}, \ldots, v_{n-1}$ be the vertices of $G$ in the order they are encountered when walking clockwise along $G$. Place each vertex $v_{i}$ at point $\left(i, \lambda\left(v_{i}\right)\right)$. Clearly none of the edges $\left(v_{i}, v_{i+1}\right)$ $(i=0,1, \ldots, n-2)$ cross each other. To avoid crossings between edge $\left(v_{0}, v_{n-1}\right)$ and the other edges we translate $v_{n-1}$ to the right until the segment connecting $v_{0}$ to $v_{n-1}$ does not cross any other segment. It is immediate to see that such a drawing is $v$-stretchable for every vertex $v$ of $G$.

Corollary 2. Let $G_{1}$ and $G_{2}$ be two matched graphs such that $G_{1}$ is a planar graph and $G_{2}$ is a cycle. Then $G_{1}$ and $G_{2}$ are matched drawable.

The drawing techniques in [10] imply the following two lemmata.

Lemma 5. Let $G$ be a caterpillar and let $v$ be a vertex of its spine. $G$ is ULP $v$-stretchable.

Lemma 6. Let $G$ be a radius-2 star and let $v$ be the center of $G$. G is ULP $v$-stretchable.

Corollary 3. Let $G_{1}$ and $G_{2}$ be two matched graphs such that $G_{1}$ is a planar graph and $G_{2}$ is a carousel graph. If each seat of $G_{2}$ is either a caterpillar with 
(a)

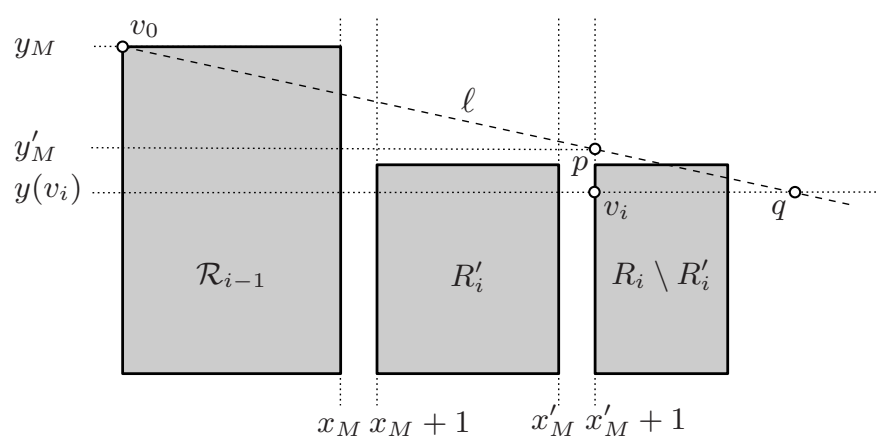

(b)

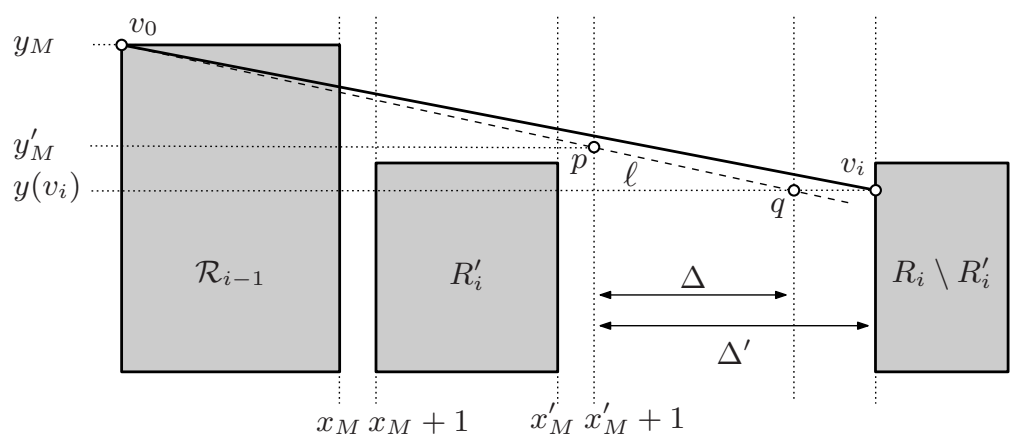

Fig. 4. Adding $\Gamma_{i}$ to $\Gamma_{2}^{i-1}$

a vertex of its spine as its hook, a radius-2 star with its center as its hook, or a cycle, then $G_{1}$ and $G_{2}$ are matched drawable.

The family of carousel graphs described by Corollary 3 contains graphs that are not ULP. For example, the graph depicted in Fig. 3 is a carousel graph with pivot $v_{2}$, the three seats are caterpillars.

\subsection{Two Trees}

Theorem 5. Any two matched trees are matched drawable.

Proof. Let $T_{1}$ and $T_{2}$ any two matched trees. We prove by construction that $T_{1}$ and $T_{2}$ are matched drawable. Let the $y$-coordinates to be used be $1, \ldots, n$, we will assign matched vertices from $T_{1}$ and $T_{2}$ consecutively to coordinates $n, 1, n-1,2, n-2,3, \ldots$ until all vertices are placed.

Let $T_{i}$ be a tree with a subset of its vertices placed. Then the maximal connected unplaced parts of $T_{i}$ are incident to one, two, or more placed vertices. We call a maximal connected unplaced part of a tree a chunk.

We maintain the following invariant for $T_{1}$ : after every odd placement, every chunk of $T_{1}$ is incident to at most two placed vertices of $T_{1}$. For $T_{2}$ we maintain a similar invariant: after every even placement, every chunk of $T_{2}$ is incident to at 

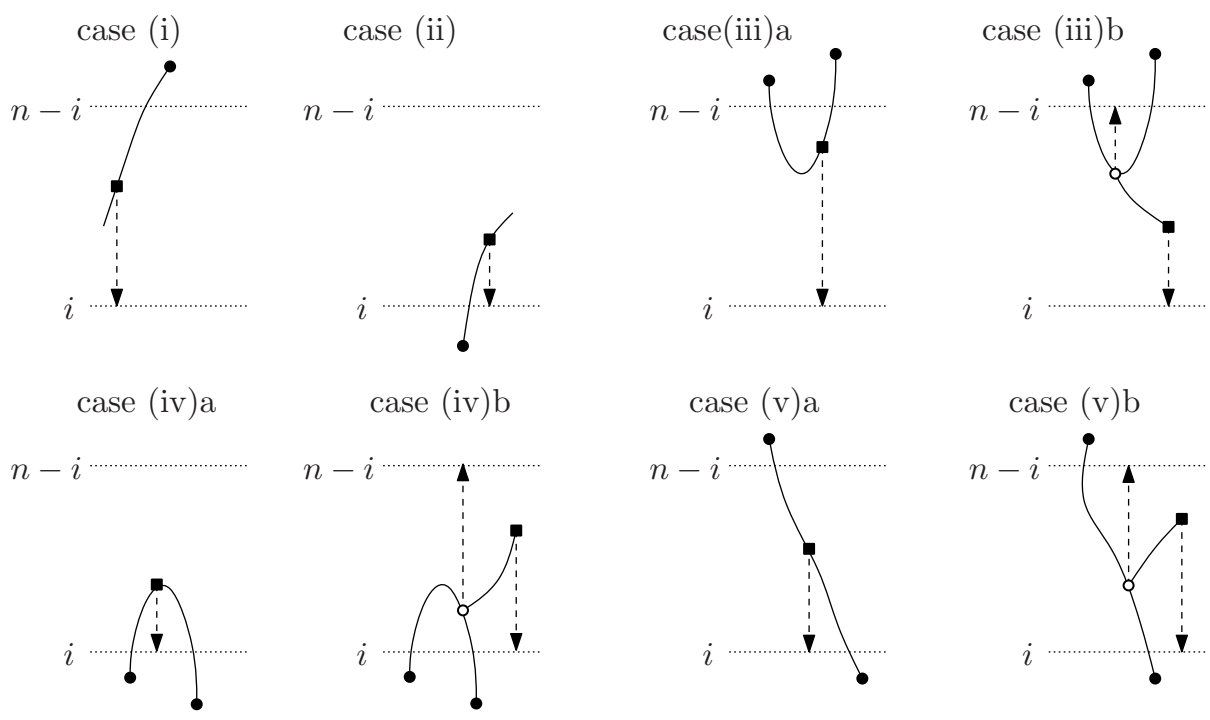

Fig. 5. The eight cases for placement at $i$ (and at $n-i$ in three cases)

most two placed vertices of $T_{2}$. We call this the topological invariant. Intuitively, tree $T_{1}$ determines which vertex is placed in odd placements at $n, n-1, n-2$, $\ldots$, and tree $T_{2}$ determines which vertex is placed in even placements at $1,2,3$, .... The other tree just follows with the matched vertex.

The topological invariants are needed for two reasons. Most importantly, they make sure that the algorithm cannot get stuck, in the sense that the placement of a vertex leads to an intersection. Secondly, they limit the number of cases that must be analyzed.

Consider $T_{1}$ after an odd placement and assume that it satisfies the invariant. Then a chunk can be one of five types: (1) it has one incident placed vertex at a high coordinate; (2) it has one incident placed vertex at a low coordinate; (3) it has two incident placed vertices at high coordinates; (4) it has two incident placed vertices at low coordinates; (5) it has one incident placed vertex at a high coordinate and one incident placed vertex at a low coordinate.

An even placement (at the bottom) may cause violation of the invariant for $T_{1}$ unless the next odd placement restores it. So for the case analysis of $T_{1}$ we will consider all possibilities of an even placement and the corresponding odd placement. If the even placement is at $i$, then the next odd placement is at $n-i$. There are eight cases to be distinguished for an even placement at $i$; they are shown in Fig. 5. In the three (.)b cases, which vertex to place at $n-i$ is determined by the fact that the topological invariant must be restored for $T_{1}$. It is the unique vertex of $T_{1}$ where the path from the just placed vertex meets the path between the two vertices that bound the chunk. It is easy to see from the figure that in the three (.)b cases the invariant can be restored for $T_{1}$ by 

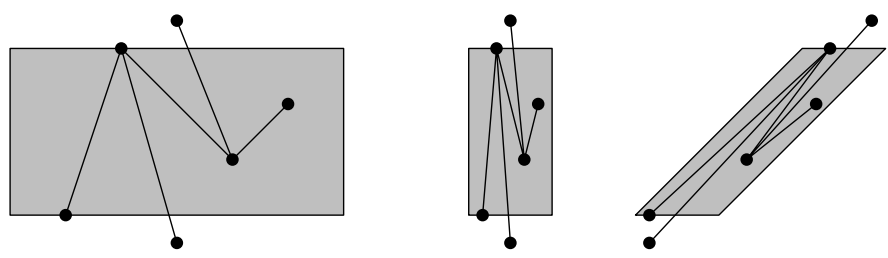

Fig. 6. Scaling and shearing a wide rectangle into a narrow parallelogram

placing this vertex at $n-i$. In the five other cases, we can assure the invariant to hold after placement at $n-i$ by choosing to place any unplaced vertex that is a neighbor of a placed vertex.

The situation is completely analogous for $T_{2}$, where an odd placement may cause a violation of the invariant if the next even placement is not chosen well.

Next we must show that there is actually space to draw the trees without crossings and with straight edges. For this we need a geometric invariant: after the placement at $n-i+1$, there is a parallelogram between the horizontal lines at $n-i$ and $i$ in which the whole chunk can be drawn without crossings and with straight edges. The parallelograms must have positive width and have an "alignment" that corresponds to the needs of the chunk. For example, for type (1) the incident placed vertex must be able to connect to any point on the far horizontal side of the parallelogram without going outside the parallelogram. It remains to show that every chunk can be drawn inside its parallelogram and that, if a chunk is split into several chunks, their resulting parallelograms are disjoint. In essence this is the case because any parallelogram can be scaled and sheared to fit, see Fig. 6. The formal statement of the geometric invariant and the remainder of the proof are omitted due to space limitations.

\section{Conclusions and Open Problems}

In this paper we introduced the concept of matched drawings, which are a natural way to draw two planar graphs whose vertex sets are matched. Since this is the first study of these drawings, many interesting and challenging open problems remain. First of all, in the light of Theorems 2 and 4, we would like to characterize the subclass of planar graphs that admit a matched drawing with any planar graph. Secondly, the drawing techniques of Theorems 4 and 5 may give rise to drawings where the area is exponential in the size of the graphs. It would be interesting to study the area requirement of matched drawings that use straightline edges. On a related note, some of our drawing techniques rely on a planar straight-line drawing of a planar graph where each vertex has a different $y$ coordinate. How big a grid is necessary to guarantee such a drawing with integer coordinates? And finally, given any two matched graphs, what is the complexity of testing whether they are matched drawable? 


\section{References}

1. Brandes, U., Erlebach, T. (eds.): Network Analysis. LNCS, vol. 3418. Springer, Heidelberg (2005)

2. Brandes, U., Erten, C., Fowler, J., Frati, F., Geyer, M., Gutwenger, C., Hong, S.-H., Kaufmann, M., Kobourov, S., Liotta, G., Mutzel, P., Symvonis, A.: Colored simultaneous geometric embeddings. In: Lin, G. (ed.) COCOON 2007. LNCS, vol. 4598, pp. 254-263. Springer, Heidelberg (2007)

3. Braß, P., Cenek, E., Duncan, C.A., Efrat, A., Erten, C., Ismailescu, D., Kobourov, S.G., Lubiw, A., Mitchell, J.S.B.: On simultaneous planar graph embeddings. Computational Geometry: Theory and Applications 36(2), 117-130 (2007)

4. Cappos, J., Estrella-Balderrama, A., Fowler, J.J., Kobourov, S.G.: Simultaneous graph embedding with bends and circular arcs. In: Kaufmann, M., Wagner, D. (eds.) GD 2006. LNCS, vol. 4372, pp. 95-107. Springer, Heidelberg (2007)

5. de Fraysseix, H., Pach, J., Pollack, R.: How to draw a planar graph on a grid. Combinatorica 10, 41-51 (1990)

6. Demetrescu, C., Di Battista, G., Finocchi, I., Liotta, G., Patrignani, M., Pizzonia, M.: Infinite trees and the future. In: Kratochvíl, J. (ed.) GD 1999. LNCS, vol. 1731, pp. 379-391. Springer, Heidelberg (1999)

7. Di Giacomo, E., Didimo, W., Grilli, L., Liotta, G.: Graph visualization techniques for web clustering engines. IEEE Transactions on Visualization and Computer Graphics 13(2), 294-304 (2007)

8. Di Giacomo, E., Liotta, G.: Simultaneous embedding of outerplanar graphs, paths, and cycles. International Journal of Computational Geometry and Applications 17(2), 139-160 (2007)

9. Erten, C., Kobourov, S.G.: Simultaneous embedding of planar graphs with few bends. Journal of Graph Algorithms and Applications 9(3), 347-364 (2005)

10. Estrella-Balderrama, A., Fowler, J.J., Kobourov, S.G.: Characterization of unlabeled level planar trees. In: Kaufmann, M., Wagner, D. (eds.) GD 2006. LNCS, vol. 4372, pp. 367-379. Springer, Heidelberg (2007)

11. Fernau, H., Kaufmann, M., Poths, M.: Comparing trees via crossing minimization. In: Proc. 25th Conf. on Foundations of Software Technology and Theoretical Computer Science, pp. 457-469 (2005)

12. Fowler, J.J., Kouborov, S.G.: Characterization of unlabeled level planar graphs. Technical Report TR06-04, Dep. of Computer Science, University of Arizona (2006)

13. Frati, F.: Embedding graphs simultaneously with fixed edges. In: Kaufmann, M., Wagner, D. (eds.) GD 2006. LNCS, vol. 4372, pp. 108-113. Springer, Heidelberg (2007)

14. Friedrich, C., Eades, P.: Graph drawing in motion. Journal of Graph Algorithms and Applications 6(3), 353-370 (2002)

15. Geyer, M., Kaufmann, M., Vrt'o, I.: Two trees which are self-intersecting when drawn simultaneously. In: Healy, P., Nikolov, N.S. (eds.) GD 2005. LNCS, vol. 3843, pp. 201-210. Springer, Heidelberg (2006)

16. Huang, M.L., Eades, P., Cohen, R.F.: WebOFDAV-navigating and visualising the web online with animated context swapping. Computer Networks and ISDN Systems 30, 638-642 (1998)

17. Misue, K., Eades, P., Lai, W., Sugiyama, K.: Layout adjustment and the mental map. Journal of Visual Languages and Computing 6(2), 183-210 (1995)

18. North, S.: Incremental layout in dynadag. In: Brandenburg, F.J. (ed.) GD 1995. LNCS, vol. 1027, pp. 409-418. Springer, Heidelberg (1996) 